\title{
Sales Bottlenecks And Their Effect On Profit
}

Mark Veltman, Rabobank, Rotterdam, Netherlands

Robert Kooij, Delft University of Technology and TNO Information and Communication Technology, Netherlands Sebastian Marban, Rabobank, Eindhoven, Netherlands

\begin{abstract}
This study introduces the term sales bottleneck, defined as a stage in a total production or service delivery process that limits sales. After analyzing the suitability of traditional methods to find sales bottlenecks, the study proposes the bottleneck accounting model as a method to determine sales bottlenecks and calculate the effect of each of these bottlenecks on profit. Analytical verification shows that this method finds all sales bottlenecks and determines the exact effect on profit. Finally, the method's effectiveness is tested in practice by means of a case study performed within the Rabobank.
\end{abstract}

Keywords: Bottleneck; Sales Bottleneck; Bottleneck Accounting; Rabobank

\section{INTRODUCTION}

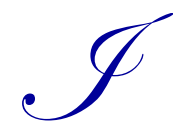

$\mathrm{n}$ a production or service process, a bottleneck is the capacity constraining stage governing the output of the entire process (Slack et al, 2010). Schmenner and Swink (1998) cite the Law of Bottlenecks, which states that the productivity of an operation is improved by eliminating or by better managing its bottlenecks. Goldratt, in his purely practitioner-oriented work, also emphasizes that a company must concentrate on its bottlenecks in order to improve overall performance (see also Goldratt \& Cox, 1984 and Fritzsch, 1998).

In recent years, many bottleneck detection methods have been presented in the literature which focus on the factory floor and only analyze the manufacturing stage, see for example Kuo et al. (1996); Roser et al. (2003); Ching et al. (2008); Li et al. (2009); Wang et al. (2012); and Xie and Li (2012). These methods do not analyze the whole production or service delivery process, which starts with an initial stage such as procurement and ultimately ends with a final stage such as sales. Because of this limited view, a bottleneck, which actually limits sales and profit but which occurs before or after the manufacturing stage, might be missed, costing the company time and money looking for a bottleneck in the wrong places.

In principle, a bottleneck affecting sales and profit can occur at any stage of the total production or service delivery process. Examples can be found during the purchasing stage, if insufficient raw materials are bought, resulting in too few products manufactured and sold; the manufacturing stage, if production levels are too low; or the sales stage, if too few clients can be persuaded to buy the products. Therefore, every stage of the total process must be analyzed. This study investigates how a company can detect the bottleneck that limits sales by analyzing the whole production or service delivery process. To do this, we consider the number of products that are generated and sold as the output of the total production or service delivery process. We introduce the term sales bottleneck, defined as a stage in the entire process that limits sales.

The negative effect that a sales bottleneck has on profit demonstrates the urgent need to eliminate the sales bottleneck. Therefore, a sales bottleneck with less impact on profit is given a lower priority than a sales bottleneck that has a drastic effect on profit. In order to determine the priority of each sales bottleneck, we also examine how a company can determine what effect each sales bottleneck has on profit. 
The fundamental contribution of this study is the proposal of a method that finds the bottleneck limiting sales and determines the effect that such a sales bottleneck has on profit. We refer to this method as bottleneck accounting. Our method is distinguished from those in previous work because to the best of our knowledge, no other method analyzes all stages of the production or service delivery process and simultaneously determines the effect a bottleneck has on profit.

The remainder of the paper is organized as follows. Section 2 investigates the extent to which sales bottlenecks can be found and its effect on profit can be determined using well-known bottleneck detection methods. Section 3 introduces the bottleneck accounting method. Analytical verification demonstrates that all sales bottlenecks can be found and their exact effect on profit can be determined with this method. Next, in Section 4 we describe the real-life implementation of the bottleneck accounting method within Rabobank, validating the effectiveness of the method in practice. Finally, Section 5 offers conclusions and suggestions for further research.

\section{EXISTING BOTTLENECK DETECTION METHODS}

In this section, we describe the most common bottleneck detection methods and investigate their effectiveness in finding sales bottlenecks. Further, we study whether these methods can be used to determine the effect of each sales bottleneck on profit.

\subsection{Queue Length}

Lawrence and Buss (1994) and Pollett (2000) postulate that the machine in front of which the longest queue occurs is the bottleneck. The main disadvantage of this method is that it may yield misleading results. That is, the longest queue might occur before a machine or workstation that received a lot of unfinished products or parts as input, but does not limit sales. Consider, for example, Company $\mathrm{Q}$ and its production process during May as illustrated in Figure 1. After the procurement of raw materials, the units of (unfinished) products first passes machine 3, then machine 2, and finally, machine 1. At the beginning of May (see A), no queues exist. During the month, the output of the purchasing department, expressed as units of a product, amounted to 400 units; the output of machine 3 was also 400 units; the output of machine 2 amounted to 100 units and the output of machine 1 was 50 units. As a result, at the end of May (see B) the queue length before machine 2 was 300 units and the queue length before machine 1 was 50 units. Hence, the longest queue occurred before machine 2, although machine 2 is not the bottleneck governing sales. Machine 1 is the bottleneck limiting the output of the total production process to only 50 units. Even if machine 2 would have processed more than 100 units, the output of machine 1 would still have been 50 units.

A:
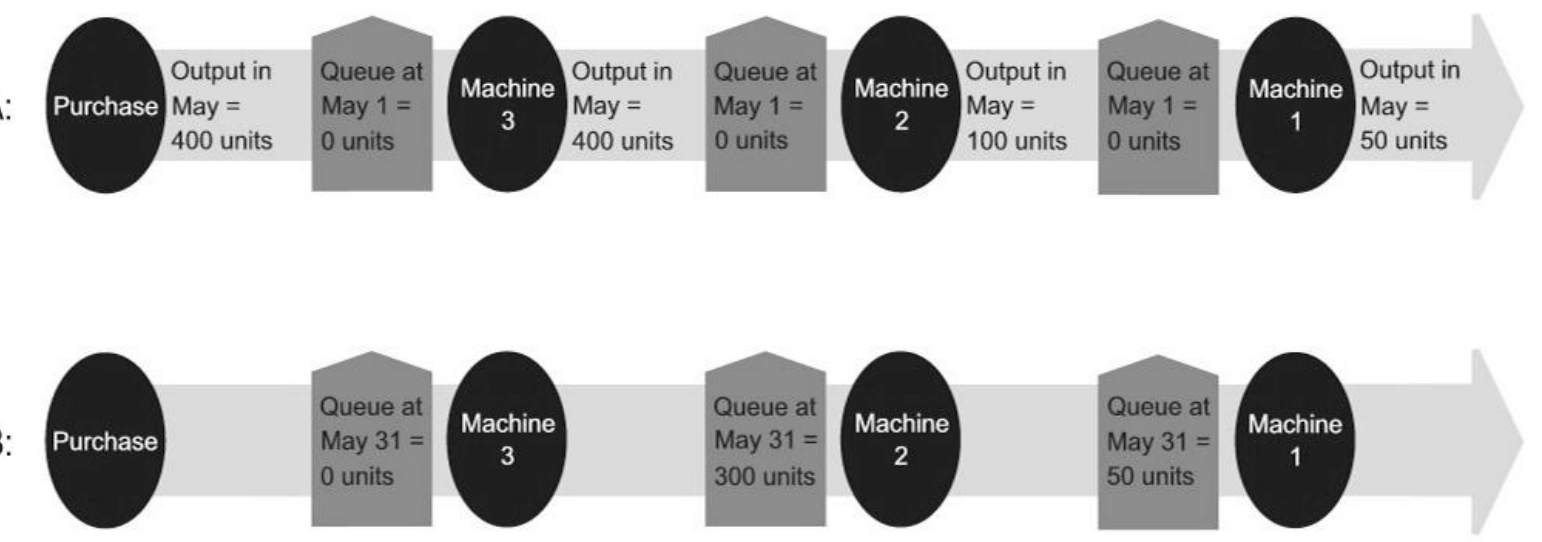

Figure 1: Queue Length, Fictitious Company Q 
Another disadvantage of this method is that, by definition, during the entire production process, no queue occurs before the initial stage, although this stage can indeed be a sales bottleneck if, for example, not enough raw materials are purchased to satisfy the demand for products. Finally, this method does not calculate the effect that a bottleneck has on profit.

\subsection{Maximal Production Capacity}

Other methods detect bottlenecks based on the maximal production capacity of production factors. On this basis, and with the aid of a technique such as linear programming (Dantzig, 1965), a company can calculate how to achieve an optimal sales volume in a certain period by optimizing the deployment of the production factors. A company can then determine which production factors are bottlenecks in the optimal situation, limiting sales volume.

A disadvantage of this approach is that the maximal production capacity of each production factor is assumed to be a known constant. However in real applications, this assumption is seldom satisfied precisely (Hillier \& Lieberman, 2005). Our case study as performed within the Rabobank, for instance, shows that the maximal production capacities of almost all factors at Rabobank are not known constants. As a result, not all production factors can be taken into account (only the known constants), and therefore, a sales bottleneck could be missed.

\subsection{Processing Times And Utilization}

Krajewski et al. (2010) and Cachon and Terwiesch (2009) describe methods that detect bottlenecks based on the utilization of production factors. First, the processing time of a production factor, also termed activity time, is defined as the average time that a production factor takes to process one or more units of a product. Next, the workload of every production factor is determined based on its average processing time and the total demand for products during period $t$. Next, the utilization of every production factor is calculated by dividing its workload by the total available time of the production factor during period $t$. The production factor with the highest utilization is then designated as the bottleneck.

A disadvantage of this method is that it assumes that a production factor can only produce one type of product at any one time. However, in practice, many production factors process multiple product types simultaneously. Some examples include an account manager working on a sales order of 100,000 units of product A and 20,000 units of product $\mathrm{B}$, a buying department purchasing raw materials for 10 different types of products during a single telephone call, and a shipping department transporting several types of products simultaneously. For these and other production factors processing multiple products simultaneously, this methodology cannot be utilized because it is not possible to unambiguously determine their processing time per product. As a result, not all stages may be analyzed and a sales bottleneck may be missed.

\subsection{Active And Inactive Periods}

Roser et al. (2001) describe a method using active and inactive periods of machines in use to find bottlenecks. The machine with the longest average active period is considered the bottleneck. The shifting bottleneck detection method, as described by Roser et al. (2003), measures how long a machine is active without interruption. In this method, the machine with the longest non-interrupted active period is designated the bottleneck.

These methods focus on the factory floor and have the disadvantage of not considering many production factors outside the factory floor, as it is not always possible to determine active and inactive periods. For example, is a relationship manager active or inactive while en route to a new client? Is a purchasing manager active or inactive during consultations? Therefore, there is a good chance that not all stages of the total production or service delivery process can be analyzed, and consequently, a sales bottleneck is missed using the active and inactive periods of production factors. In addition, this method does not calculate the influence a bottleneck has on profit. 


\subsection{Blockage And Starvation}

Among others, Ching et al. (2008), Kuo et al. (1996), and Li et al. (2009) determine bottlenecks based on the time a machine is inactive because it is waiting for the arrival of parts, a period called starvation, or for their removal, a period called blockage.

As with previous methods, this method focuses on factory production and cannot be used to analyze all production factors. This is because this method assumes that a production factor waiting for the arrival of parts or for the removal of processed parts will be inactive. However, many production factors outside the factory floor will remain active if they have to wait for the arrival of parts or for their removal and will therefore never experience a period of starvation or blockage. For instance, a sales representative waiting for the arrival of goods for transaction $x$ while working on transaction $y$ is not inactive and will not experience a period of starvation. A purchasing department that procured raw materials A, which are still waiting to be processed, is not inactive, as it is involved in ordering raw materials B and therefore, does not experience a period of blockage. In addition, these methods do not calculate the effect a bottleneck has on profit.

\section{THE BOTTLENECK ACCOUNTING METHOD}

Since the traditional methods are not suitable to determine the sales bottlenecks and their effect on profit, we introduce an alternative method, hereafter referred to as bottleneck accounting.

\subsection{All Stages In The Total Production Process}

In principle, a bottleneck that limits sales can occur in any stage of the total production or service delivery process; therefore, the bottleneck accounting method must analyze each stage. To do this, it first defines the components of the product or service and then determines all stages each component passes through in the entire process, including stages for which no queues arise and for which the active and inactive periods, the maximal production capacity, the processing times or the periods of starvation and blockage cannot be determined.

A fictitious company, Company Z, is illustrated in Figure 2. This company makes and sells tables that consist of two components: a wooden tabletop and a steel support. The first component, the tabletop, passes through a total of five stages: the wood purchasing stage, the sawing stage, the polishing stage, the assembly stage and the sales stage. The second component, the steel support frame, passes through four stages: the steel purchasing stage, the rolling stage, the assembly stage, and the sales stage.

To identify a stage, we use the following notation throughout this paper:

$S_{g, c, t} \quad$ The name of stage $g$ in the production of component $c$ during period $t$, where

g stage index, $g=1,2, \ldots, n$;

$c \quad$ component index, $c=1,2, \ldots, m$;

$t \quad$ time period index, $t=1,2, \ldots, T$. 


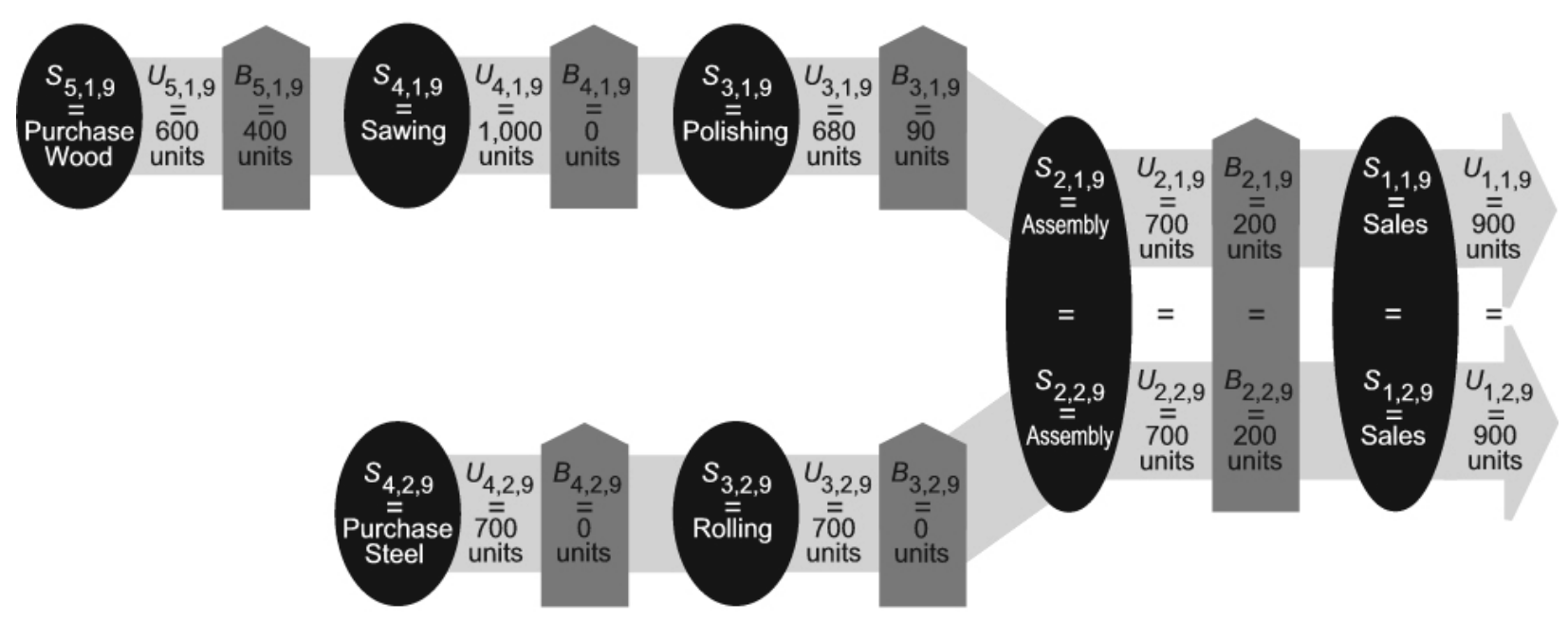

Figure 2: The Bottleneck Accounting Method For Company Z In September $(T=9)$

Note that the stages occur from fifth to first, with the last stage processing component $c$ shown as $S_{1, c, t}$. It is possible that a specific stage in the total production process of a component is the same as a stage in the total production process of another component. In Company $\mathrm{Z}$, for example, the assembly stage of the wooden tabletop, stage $S_{2,1,9}$ refers to the same stage as the assembly stage of the steel support, $S_{2,2,9}$. This also holds for the last stage, where the tabletop and steel support are sold together as a complete product.

\subsection{Output And Initial Inventory Of Work In Progress}

The output of a stage indicates the number of units of a component that have passed through this stage during a period. This output may be higher in one period than in another period.

To denote the output of a stage and the initial inventory of work in progress directly downstream, we use the following notations:

$U_{g, c, t} \quad$ The output of stage $S_{g, c, t}$ that is, the number of units of component $c$ that pass through stage $S_{g, c, t}$ during period $t$.

$B_{g, c, t} \quad$ The initial inventory of work in progress directly downstream from stage $S_{g, c, t}$, that is, the number of units of component $c$ that have already passed through stage $S_{g, c, t}$ before period $t$ but have not yet passed the stage immediately downstream from stage $S_{g, c, t}$ and are thus available at the beginning of period $t$.

Figure 2 shows all the stages of the total production process of Company Z, including the output and initial inventory of each stage. Consider, for example, the polishing stage in which 680 tabletops are polished in September. Hence, the output of the polishing stage was 680 tabletops. Next, consider the wood purchasing stage. In September, 4 tree trunks were purchased, from which 600 tabletops could be manufactured. The output of the wood purchasing stage therefore amounted to 600 tabletops. The initial inventory of wood that had been purchased but not yet sawn amounted to 400 units of the component 'table top'. This was because an initial inventory of wood was available for a total of 400 table tops.

If a specific stage in the total production process of a component is the same as a stage in the total production process of another component, the output and initial inventory of the work in progress of both stages are equal. For example, in Company Z, the output of stage $S_{2,1,9}$, shown as $U_{2,1,9}$, is equal to the output of stage $S_{2,2,9}$, shown as $U_{2,2,9}$, and the initial inventory of stage $S_{2,1,9}$, shown as $B_{2,1,9}$, is equal to the initial inventory of $S_{2,2,9}$, shown as $B_{2,2,9}$. 
The bottleneck accounting method makes the following assumption:

Assumption: A unit of every product that is sold contains one unit of every component.

A table sold by Company Z, for instance, consists of one wooden tabletop and one steel support. It may be that a complete product includes more units of a specific part. A complete table, such as is sold by Company Z, for example, has four steel table legs. In the bottleneck accounting method, we consider these four table legs as one set, a single unit of the steel support component.

\subsection{Stage Inputs}

The input of a stage consists of the units of a component that a previous stage has already processed and that must be further processed by the stage in question. The input of a stage thus contains the output of a previous stage. Further, inputs consist of units of a component present in the initial inventory of work in progress that is situated between the stage in question and the previous stage. For all $g, c$, and $t$, the input for stage $S_{g, c, t}$ is denoted as $I_{g, c, t}$ and is equal to

$I_{g, c, t}=U_{g+1, c, t}+B_{g+1, c, t}$

One stage may also receive input from two different stages. The assembly stage in Figure 2, for example, receives input from both the polishing stage and the rolling stage. The assembly stage can only process the input of a unit of a component if it can combine this with a unit of the other component. In general, suppose that stage $S_{g, c, t}$ receives the input of $m$ previous stages. Then, for all $g, c$, and $t$, the input that stage $S_{g, c, t}$ receives can be calculated as

$I_{g, c, t}=\min \left\{\left(U_{g+1,1, t}+B_{g+1,1, t}\right), \ldots,\left(U_{g+1, m, t}+B_{g+1, m, t}\right)\right\}$

For example, in September, the assembly stage of Company Z could combine only 700 out of $770(=680+$ 90) polished tabletops with rolled supports. Hence, the input of the assembly stage amounted to 700 units. In Table 1 , the input of each stage in September is calculated using Equations (1) and (2).

Table 1: Stage Inputs

\begin{tabular}{|c|c|c|c|c|c|c|c|}
\hline & $\begin{array}{c}\text { Wood } \\
\text { Purchase }\end{array}$ & Sawing & Polishing & Assembly & Sales & $\begin{array}{c}\text { Steel } \\
\text { Purchase }\end{array}$ & Rolling \\
\hline$I_{g, c, t}$ & n.a. & 1,000 & 1,000 & 700 & 900 & n.a. & 700 \\
\hline
\end{tabular}

\subsection{Actual Sales}

During a specific period, a stage cannot process more units of a component than it receives as input. Thus, the output of a stage during period $t$ can never be higher than its input during period $t$. This gives for all $g, c$, and $t$,

$U_{g, c, t} \leq I_{g, c t}$

The combination of Equations (1) and (3) gives

$U_{g, c, t} \leq U_{g+1, c, t}+B_{g+1, c, t}$

The final output of the total production or service delivery process $V_{t}$, is for all $c$ and $t$ equal to the output of the final stage:

$V_{t}=U_{1, c, t}$

After the final stage, there is no initial work in progress. That is, for all $c$ and $t$, 
$B_{l, c, t}=0$

By combining Equations (4), (5), and (6), we obtain

$V_{t} \leq U_{2, c, t}+B_{2, c, t}+B_{1, c, t}$

By combining Equations (4) and (7) recursively, we obtain the following theorem.

Theorem 1. For all $g, c$, and $t$,

$V_{t} \leq U_{g, c, t}+\sum_{b=1}^{g} B_{b, c, t}$

The final sales are thus limited by the output of stage $S_{g, c, t}$ plus the initial work in progress in all stages downstream from $S_{g, c, t}$. This will also be referred to as the available production $A_{g, c, t}$ of stage $S_{g, c, t}$, since it consists of the units of component $c$ that have passed through $S_{g, c, t}$ and that are available at the beginning of period $t$ or will become available during period $t$ as an output of $S_{g, c, t}$. That is, for all $g, c$, and $t$,

$A_{g, c, t}=U_{g, c, t}+\sum_{b=1}^{g} B_{b, c, t}$

Combining Equations (8) and (9) gives

$V_{t} \leq A_{g, c, t}$

Since a company cannot sell more than it produces, the sales of a company cannot be higher than the available production of any stage, so the actual sales in period $t$ will also be equal to the lowest available production of all stages in period $t$. This brings us to the following theorem.

Theorem 2. For all $g, c$, and $t$,

$V_{t}=\min \left\{A_{1,1, t}, \ldots, A_{n, m, t}\right\}$

Proof: Let $t \in\{1,2, \ldots, T\}$. We define

$A_{\text {min }, t}=\min \left\{A_{1,1, t}, \ldots, A_{n, m, t}\right\}$

Proving Theorem 2 is the same as showing that Equations (13) and (14) hold:

$V_{t} \leq A_{\text {min, }}$

$V_{t} \geq A_{\text {min, } t}$

Equation (13) follows directly from Equation (10), which states that $V_{t}$ is less than or equal to the available production of all stages in period $t$. Hence, it is also less than or equal to the lowest available production.

Equation (14) can be shown as follows:

$A_{\min , t}$ is the lowest available production of all stages in period $t$, such that for all $c$ and $t$,

$A_{\min , t} \leq A_{1, c, t}$

By combining Equation (15) with Equations (6) and (9), we obtain

$A_{\text {min,t }} \leq U_{1, c, t}$ 
Combining Equation (16) with Equation (5) then gives

$A_{m i n, t} \leq U_{1, c, t}=V_{t}$

Hence,

$A_{\min , t} \leq V_{t}$

demonstrating that Equation (14) holds.

By proving Equations (13) and (14), we also prove Theorem 2.

\subsection{Bottlenecks}

We defined a sales bottleneck as a stage in the total production process that limits sales. Theorem 2 states that the actual sales are limited to the lowest available production of all stages of the entire process. Therefore, in order to detect the sales bottlenecks, the bottleneck accounting method focuses on the stages with the lowest available production.

If a stage has the lowest available production, this production might have originated during the stage in question itself. This stage then caused the actual sales to be limited to the lowest available production and is a sales bottleneck. It may also happen that during one stage the lowest available production occurs because it received too little input. As stated in Equation (3), a stage cannot process more components than it has received as input. Even if a stage has an enormous production capacity its output remains equal to its input. In this case, low production is not caused by the stage in question but by a previous stage. Thus not this stage but a previous stage is the sales bottleneck.

In short, stage $S_{g, c, t}$ is only a sales bottleneck during time period $t$ if it has the lowest available production and if its output $U_{g, c, t}$ is not equal to its input $I_{g, c, t}$. A formal definition of the sales bottleneck can therefore be given as follows:

Definition: For all $g, c$, and $t$, stage $S_{g, c, t}$ is a sales bottleneck if

$A_{g, c, t}=A_{m i n, t}$ and $U_{g, c, t} \neq I_{g, c, t}$

Using the definition above, we can determine the sales bottlenecks of Company Z, as shown in Table 2. This table shows that during September, the assembly, sales, steel purchasing, and rolling stages had the lowest available production. However, the assembly, sales, and rolling stages received insufficient input so are not sales bottlenecks.

Table 2: Determination Of Sales Bottlenecks

\begin{tabular}{|l|c|c|c|c|c|c|c|}
\hline \multicolumn{1}{|c|}{$\mathbf{1}$} & $\mathbf{2}$ & $\mathbf{3}$ & $\mathbf{4 = 2 + 3}$ & $\mathbf{5}$ & $\mathbf{6}$ & $\mathbf{7}$ & \multicolumn{1}{c|}{$S_{g, c, t}$} \\
\hline$U_{g, c, t}$ & $\sum_{b=1}^{g} B_{b, c, t}$ & $U_{g, c, t}+\sum_{b=1}^{g} B_{b, c, t}$ & $I_{g, c, t}$ & $A_{g, c, t}=A_{m i n, t}$ & $U_{g, c, t} \neq I_{g, c, t}$ & $\begin{array}{c}\text { Sales } \\
\text { Bottleneck }\end{array}$ \\
\hline $\begin{array}{l}\text { Wood } \\
\text { Purchasing }\end{array}$ & 600 & 690 & 1,290 & n.a. & No & Yes & No \\
\hline Sawing & 1,000 & 290 & 1,290 & 1,000 & No & No & No \\
\hline Polishing & 680 & 290 & 970 & 1,000 & No & Yes & No \\
\hline Assembly & 700 & 200 & 900 & 700 & Yes & No & No \\
\hline Sales & 900 & 0 & 900 & 900 & Yes & No & No \\
\hline $\begin{array}{l}\text { Steel } \\
\text { Purchasing }\end{array}$ & 700 & 200 & 900 & n.a. & Yes & Yes & Yes \\
\hline Rolling & 700 & 200 & 900 & 700 & Yes & No & No \\
\hline
\end{tabular}

Copyright by author(s); CC-BY 
The steel purchasing stage also had the lowest available production, while its output was not limited by its input. The lowest available production, therefore, originated from the steel purchasing stage itself and hence, formed the sales bottleneck in September.

From Equation (10), it follows that the sales cannot be higher than the available production of any stage. Thus, sales are also limited by the available production of the stage that has the second lowest available production, denoted as $A_{d, t}$ :

$V_{t} \leq A_{d, t}$

However, owing to the occurrence of the sales bottleneck, sales are not limited to $A_{d, t}$ but are limited to the lowest available production. We indicate the effect of the sales bottleneck as $E_{f, t}$ :

$E_{f, t}=A_{\min , t}-A_{d, t}$

For example, Company Z's sales were already limited because an available production of 970 units had arisen during the polishing stage, the second lowest available production. The steel purchasing bottleneck limited the sales to only 900 tables. Using Equation (21), the effect of the sales bottleneck can therefore be calculated as a loss of 70 units. See Figure 3 for a graphical depiction of this effect.

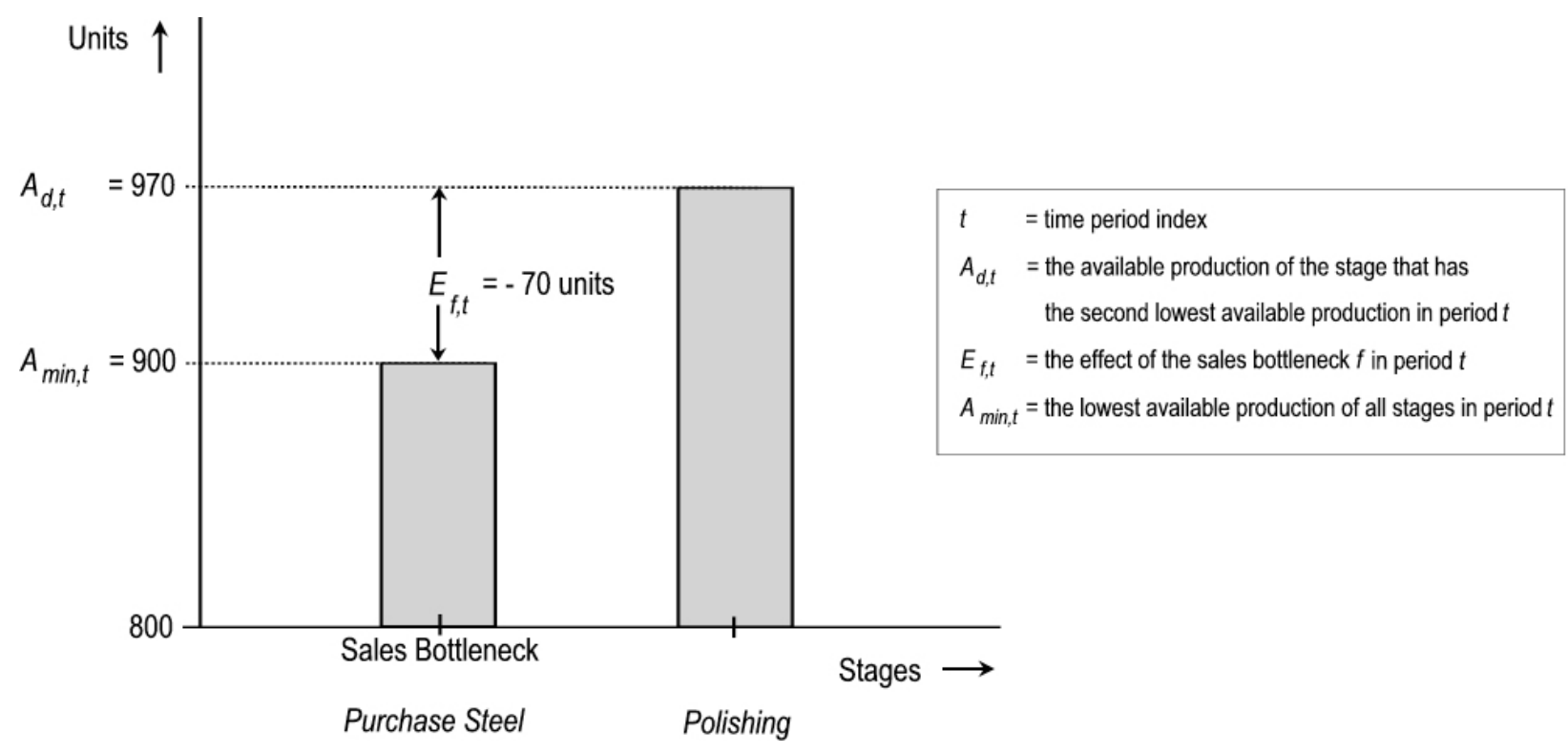

Figure 3: The Effect Of The Sales Bottleneck Of Company Z

\subsection{The Effect Of A Sales Bottleneck On Profit}

The previous subsection determined the missed sales resulting from a sales bottleneck, the effect of a sales bottleneck. In this section, we calculate the effect of a sales bottleneck on the profit of a company.

The contribution margin of a production unit of a product, denoted as $C M$, shows the profit from the sale of a unit of that product. The contribution margin of a unit is equal to the selling price per unit minus the production and sales costs for an additional unit of that product (Horngren \& Foster, 1987). 
The result of a sales bottleneck is that fewer products are sold, and so the target contribution margin of a production unit is missed. Hence, the economic effect of a sales bottleneck, $E E_{f, t}$, can be calculated as the effect of the sales bottleneck, $E_{f, t}$, multiplied by the contribution margin of a product unit:

$E E_{f, t}=E_{f, t} \cdot C M$

For example, in September, the contribution margin that Company $\mathrm{Z}$ achieved per table amounted to $\$ 300$. According to Equation (22), the economic effect of the steel purchasing bottleneck was a loss of $\$ 21,000$.

\subsection{Management Insights}

The bottleneck accounting method detects the bottlenecks that actually limit the sales, regardless of the stage in which these occur. Moreover, the bottleneck accounting method calculates the lost profit from these bottlenecks. This economic effect and the frequency of a sales bottleneck show the urgent need to eliminate a sales bottleneck. For instance, a sales bottleneck that has less effect on profits receives a lower priority than a sales bottleneck with a significant effect on profit and that arises frequently. This way the bottleneck accounting method provides relevant information that helps managers prioritize and tackle missed sales opportunities.

\section{CASE STUDY WITHIN RABOBANK}

Rabobank $^{1}$ serves around 10 million clients internationally. Rabobank's various business units, including the Regional Southwest Netherlands team (RSWN), focus their activities on large business clients selling products related to cash management services, financing, treasury, and insurance. In 2011 and 2012, the RSWN was confronted by lower-than-targeted sales. The management at the RSWN, therefore, wanted to find the sales bottlenecks and determine the effect of each on profit. This process would enable management to prioritize and manage the causes of low sales.

To determine the sales bottlenecks and their effect on profit, we interviewed eight account managers and three members of the management team. We investigated the degree to which the sales bottlenecks could be found using traditional methods and whether the bottlenecks and economic effect could be determined using the bottleneck accounting method.

\subsection{Bottleneck Detection At Rabobank Using Traditional Methods}

First, we investigated whether the sales bottlenecks could be determined on the basis of the length of the queues. In fact in RSWN it is also true that here can never be a queue before the initial stage of the total process, while this initial stage can indeed be a sales bottleneck. The initial stage in the production of a specific financing product, for example, is that a client submits a request for a loan to RSWN. A stock of work in progress will never originate before this initial stage although this stage can indeed be the sales bottleneck if few clients submit a request for a loan.

During the interviews, we also investigated the degree to which sales bottlenecks could be determined based on the maximal production capacity of the production factors. Indeed, for many production factors at the RSWN, the maximal production capacity is not a known constant. During some weeks, an insurance advisor can succeed in closing 10 agreements, while during others, the same insurance advisor may sell nothing. The same holds true for account managers. Therefore, the RSWN could not unambiguously determine the maximal production capacity of the production factors.

We also investigated to what extent the sales bottlenecks could be determined based on processing times and the utilization of production factors. However, many production factors at the RSWN process different types of products simultaneously. For example, it was not possible to determine an unambiguous processing time per type of

${ }^{1}$ The exact data was modified to exclude sensitive competitive information. 
product for an analyst who handles requests for three different products—a bank guarantee, a loan, and creditsimultaneously from one credit application.

Finally, we investigated the degree to which the sales bottlenecks could be detected based on active and inactive production periods or based upon periods of blockages or starvation. At the RSWN, the employees are the most important production factors, and for these production factors, it was particularly difficult to determine the active periods, the inactive periods, and the periods of blockage and starvation. For example, it was not possible to determine unambiguously whether an account manager engaged in a discussion with a client was active or not. It was also difficult to determine whether a financing analyst was blocked while the completed analysis was awaiting approval from the credit committee and during which period this analyst worked on another request. In fact, it was not possible to determine active periods, inactive periods, and periods of blockage or starvation of the production factors in any stage of the total production process.

From the interviews, the traditional methods could not determine sales bottlenecks. For all of these methods, the key information could not be determined or estimated with reasonable accuracy.

\subsection{The Bottleneck Accounting Method At Rabobank}

To implement the bottleneck accounting method at the RSWN, we mapped out the entire process for each product, first by determining the constituent components and then the stages each component passed through during the total process. For example, for loan product $q$, we established that it consisted of three intangible components: a request for a loan by the client, the funding for the requested loan, and a guarantee from the Dutch Government. Figure 4 shows all the stages of the process for the generation of loan product $q$.

The client request is first received during a meeting with the client (Call Client). Next, the client provides the relevant information (Collect Data). The client request is analyzed using this information (Analysis). Next, the loan grant must be approved by the credit commission (Approve). After approval, the client's request is translated into a term sheet (Offer). Finally, a loan $q$ is sold once the client accepts the proposal (Sales).

The second component, funding for the requested loan, is first purchased from the money market (Funding). In the financing proposal, it is recommended that the acquired funds are made available to the client (Offer). By accepting the proposal, the client accepts that the funds are actually lent to him (Sales).

The state guarantee forms the third component, and the Rabobank Group requests a maximum number (Quota) of state guarantees per period. A portion of these requests is assigned to the RSWN (Adjudge). In the loan offer, it is proposed that the guarantee of the Dutch State will be incorporated in the loan structure (Offer). At the sales stage (Sales), the client accepts that this guarantee actually is a component of the loan.

For each stage in the product generation process, we have determined the output and the inventories during the period analyzed, referred to as Period 7. In Figure 4, for example, the output and the initial stock are shown for loan $q$, expressed as the number of units of the component concerned. 

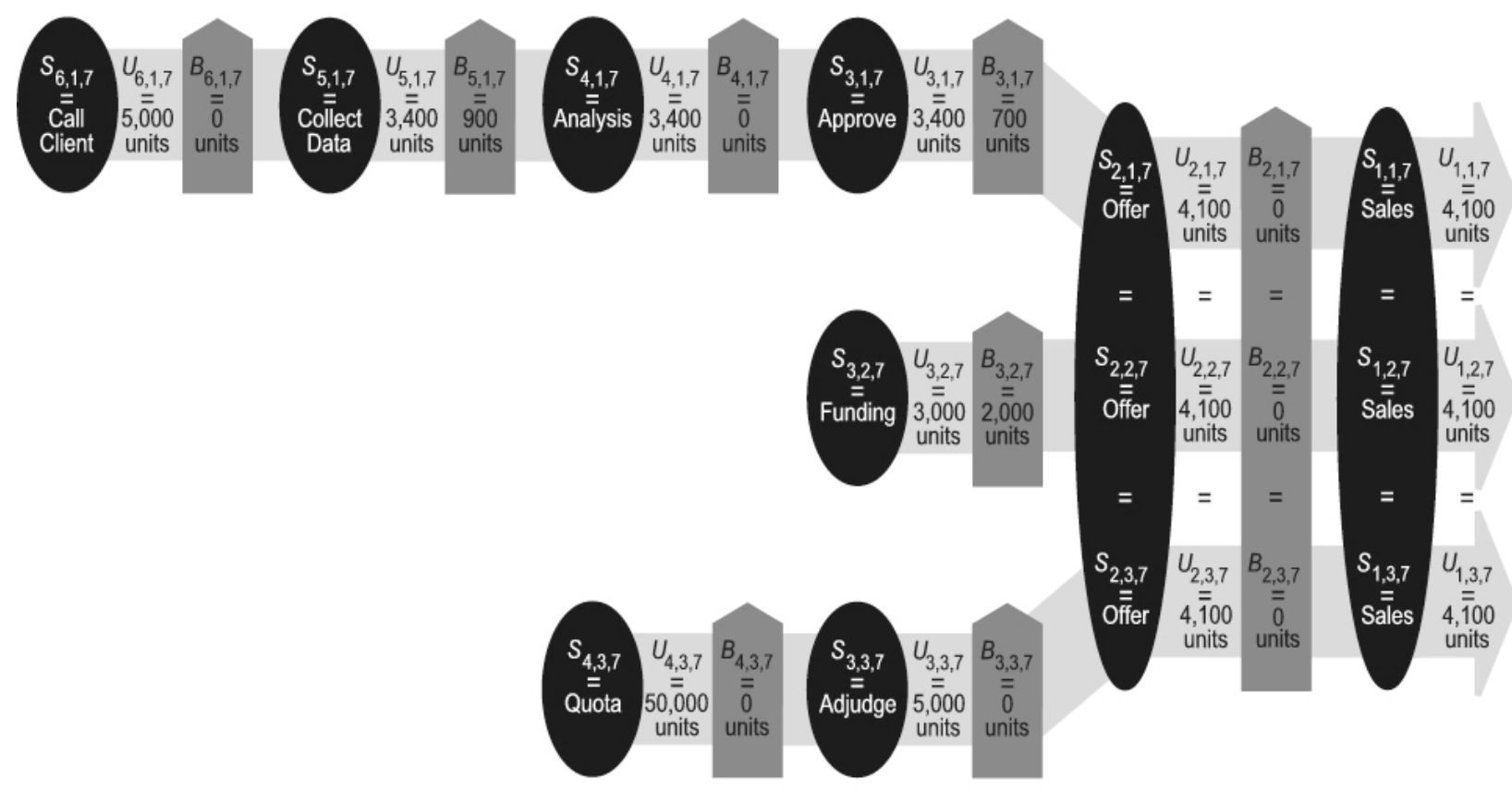

Figure 4: The Bottleneck Accounting Method At Rabobank

A unit of the component loan request consists of a request for a loan of $€ \mathrm{x}$, a unit of the component funding consists of $€ x$ funding, and a unit of the guarantee component consists of a guarantee for the loan of $€ x$. Finally, as assumed in the bottleneck accounting method, one unit of a complete loan $q$ comprises one unit of each component and concerns a loan of $€ x$. The final value of a loan $q$ provided to a specific client can consist of multiple units of loan $q$.

For example, the output of the call client stage during Period 7 was 5,000 units because the RSWN was requested to provide 5,000 units of loan $q$. The output of the approval stage was 3,400 units because the credit committee approved the grant of a total of 3,400 units of loan $q$ in Period 7. At the beginning of Period 7, there was an initial work in progress inventory that consisted of data collected for the financing of 900 units of loan $q$, the approval for the grant of 700 units of loan $q$, and the funding for a total of 2,000 units of loan $q$.

Table 3 shows how the available production for each stage in the generation of loan $q$ was determined during Period 7 and how, using Equation (19), the sales bottlenecks were determined.

Table 3: Detecting Bottlenecks In Loan $q$ Generation

\begin{tabular}{|l|c|c|c|c|c|c|c|}
\hline \multicolumn{1}{|c|}{$\mathbf{1}$} & $\mathbf{2}$ & $\mathbf{3}$ & $\mathbf{4 = 2 + 3}$ & $\mathbf{5}$ & $\mathbf{6}$ & $\mathbf{7}$ & $\mathbf{8}$ \\
\hline \multicolumn{1}{|c|}{$S_{g, c, t}$} & $U_{g, c, t}$ & $\sum_{b=1}^{g} B_{b, c, t}$ & $U_{g, c, t}+\sum_{b=1}^{g} B_{b, c, t}$ & $I_{g, c, t}$ & $\begin{array}{c}A_{g, c, t}= \\
\min \left\{A_{\left.1,1, t, \ldots, A_{n, m, t}\right\}}\right.\end{array}$ & $U_{g, c, t} \neq I_{g, c, t}$ & $\begin{array}{c}\text { Sales } \\
\text { Bottleneck }\end{array}$ \\
\hline Call Client & 5,000 & 1,600 & 6,600 & n.a. & No & Yes & No \\
\hline Collect Data & 3,400 & 1,600 & 5,000 & 5,000 & No & Yes & No \\
\hline Analysis & 3,400 & 700 & 4,100 & 4,300 & Yes & Yes & Yes \\
\hline Approve & 3,400 & 700 & 4,100 & 3,400 & Yes & No & No \\
\hline Offer & 4,100 & 0 & 4,100 & 4,100 & Yes & No & No \\
\hline Sales & 4,100 & 0 & 4,100 & 4,100 & Yes & No & No \\
\hline Funding & 3,000 & 2,000 & 5,000 & n.a. & No & Yes & No \\
\hline Quota & 50,000 & 0 & 50,000 & n.a. & No & Yes & No \\
\hline Adjudge & 5,000 & 0 & 5,000 & 50,000 & No & Yes & No \\
\hline
\end{tabular}


We used the above method to detect sales bottlenecks for all products. We determined the effects of the sales bottlenecks using Equation (21) and the economic effect using Equation (22). For example, the effect of the sales bottleneck in the Analysis stage that occurred in the generation of loan $q$ was calculated with Equation (21) as a loss of 900 units. The average contribution margin of one unit of loan $q$ was $€ 730$. Using Equation (22), the economic effect of the sales bottleneck was a loss of $€ 657,000$ in profit.

This method was used to calculate the effect of each sales bottleneck on profit. Using this information, the RSWN's management could prioritize and address the bottlenecks that had caused lower sales than forecasted for Period 7. Among other factors, the RSWN's management prioritized the Analysis bottleneck for loan $q$ in order to prevent a potentially missed profit of $€ 657,000$ for Period 8 .

The bottleneck accounting method helped the management at the RSWN focus on the sources of low sales. After Period 7, the management focused on sales bottlenecks by improving the working methods and by investing in these stages. As a result, the RSWN realized the highest sales among all regional teams in the Netherlands in 2013.

\section{CONCLUSION}

In this study, we considered how a company with a process that generates physical products or services could determine the bottleneck that limits sales and calculate the effect a sales bottleneck has on profit. We concluded that the traditional methods to detect bottlenecks based upon queue length might yield misleading results because the longest queue might occur during a stage with no impact on sales. Moreover, a method based on queue length cannot analyze the initial stage of a total process. Methods that detect bottlenecks based on the maximal production capacity of production factors, the processing times of production factors, the active or inactive periods, or the periods of starvation and blockage cannot analyze all production factors. As a result, a sales bottleneck may be missed. We also argued that the methods which detect bottlenecks by measuring queue lengths, active periods, inactive periods, periods of blockage or periods of starvation do not calculate the effect a bottleneck has on profit.

Bottleneck accounting is a suitable method to determine sales bottlenecks and their effect on profit, regardless of the stage in which these sales bottlenecks occur. The bottleneck accounting method detects the bottlenecks based on the input and output of each stage in the total production or service delivery process and the initial inventories of work in progress. It can therefore analyze all stages of the total process and thus, detect the bottleneck that actually limits sales. The bottleneck accounting method also calculates the effect a sales bottleneck has on profit. As a result, the bottleneck accounting method enables companies to focus on solving the most urgent sales bottlenecks.

The output of a stage in the production process can vary between periods. Therefore, sales bottlenecks and their effect on profit can also vary over time. This is a limitation of the bottleneck accounting method. Further study should aid in understanding this variability. In addition, empirical research is needed to confirm that the bottleneck accounting method can be used successfully by other companies.

\section{AUTHOR INFORMATION}

Mark Veltman received his MSc degree in Economics in 1995 from the Erasmus University Rotterdam in the Netherlands. He is currently a senior consultant at the Rabobank.

Robert Kooij obtained his PhD degree in Mathematics at the Delft University of Technology in the Netherlands in 1993. He is currently a senior scientist at the Performance of Networks and Systems department of TNO and a Professor of Mathematics at the Delft University of Technology in the Netherlands

Sebastian Marban obtained his PhD degree in Operations Research at Maastricht University in the Netherlands in 2012. He is currently a financial analist at Group Risk Management of the Rabobank. 


\section{REFERENCES}

1. Cachon, G., \& Terwiesch, C. (2009). Matching supply with demand: An introduction to operations management (2nd ed.). New York: McGraw-Hill.

2. $\quad$ Ching, S., Meerkov, S. M., \& Zhang, L. (2008). Assembly systems with non-exponential machines: Throughput and bottlenecks. Nonlinear Analysis: Theory, Methods \& Applications, 69(3), 911-917.

3. Dantzig, G. B. (1965). Linear programming and extensions. Princeton: Princeton University Press.

4. Goldratt, E. M., \& Cox, J. (1984). The goal: A process of continuous improvement, New York: North River Press.

5. Fritzsch, R. B. (1998). Activity-based costing and the theory of constraints: Using time horizons to resolve two alternative concepts of product cost. Journal of Applied Business Research, 14(1), 83-90.

6. Hillier, F., \& Lieberman, G. (2005). Introduction to operations research (8th ed.) New York: McGrawHill.

7. Horngren, C. T., \& Foster, G. (1987). Cost accounting: A managerial emphasis (6th ed.). London: Prentice-Hall International, Inc.

8. Krajewski, L. J., Ritzman, L. P., \& Malhotra. M. K. (2010). Operations management: Processes and supply chains (9th ed.). New Jersey: Pearson Prentice Hall.

9. Kuo, C. T., Lim, J. T., \& Meerkov, S. M. (1996). Bottlenecks in serial production lines: A system-theoretic approach. Mathematical Problems in Engineering, 2(3), 233-276.

10. Lawrence, S. R., \& Buss, A. H. (1994). Shifting production bottlenecks: Causes, cures, and conundrums. Production and Operations Management, 3(1), 21-37.

11. Li, L., Chang, Q., \& Ni, J. (2009). Data driven bottleneck detection of manufacturing systems. International Journal of Production Research, 47(18), 5019-5036.

12. Pollett, P. K. (2000). Modelling congestion in closed queueing networks. International Transactions in Operational Research, 7(4-5), 319-330.

13. Roser, C., Nakano, M., \& Tanaka, M. (2001). A practical bottleneck detection method. Peters, B.A., Smith, J. S., Medeiros, D. J. \& Rohrer, M. W. eds. in Proceedings of the 2001 Winter Simulation Conference (pp. 949-953). IEEE Computer Society.

14. Roser, C., Nakano, M., \& Tanaka, M. (2003). Comparison of bottleneck detection methods for AGV systems. Chick, S., Sanchez, P. J., Ferrin, D. \& Morrice, D.J. eds. in Proceedings of the 2003 Winter Simulation Conference (Vol. 2, pp. 1192-1198). IEEEr Society..

15. Schmenner, R. W., \& Swink, M. L. (1998). On theory in operations management. Journal of Operations Management, 17(1), 97-113.

16. Slack, N., Chambers, S., \& Johnston, R. (2010). Operations management (6th ed.). Essex: Pearson Education.

17. Wang, J., Li, J., Arinez, J., \& Biller, S. (2012). Indicators for quality improvability and bottleneck sequence in flexible manufacturing systems with batch production. International Journal of Production Research, 50(22), 6388-6402.

18. Xie, X., \& Li, J. (2012). Modeling, analysis and continuous improvement of food production systems: A case study at a meat shaving and packaging line. Journal of Food Engineering, 113(2), 344-350. 